\title{
Diferenciación morfológica en especies del género Cheirodon (Ostariophysi: Characidae) mediante morfometría tradicional y geométrica
}

\section{Morphological differentiation in the genus Cheirodon (Ostariophysi: Characidae) using both traditional and geometric morphometrics}

\author{
Daniel Salas ${ }^{1,2}$, David Véliz ${ }^{1,2}$, Sergio Scott ${ }^{1 *}$ \\ 1Departamento de Ciencias Ecológicas, Facultad de Ciencias, Universidad de Chile, Casilla 653, Santiago, Chile. . \\ ${ }^{2}$ Instituto de Ecología y Biodiversidad, Facultad de Ciencias, Universidad de Chile, Casilla 653, Santiago, Chile. \\ *Email: sergio.scott@gmail.com
}

\begin{abstract}
RESUMEN
Los peces dulceacuícolas del género Cheirodon están representados en Chile por cinco especies: Cheirodon pisciculus, Ch. australe, Ch. galusdae, Ch. kiliani y Ch. interruptus, esta última introducida accidentalmente desde Argentina. La escasa diferenciación morfológica, además de la asociación simpátrica de Ch. pisciculus con Ch. interruptus y de Ch. galusdae con Ch. australe en parte de sus distribuciones, dificultan su identificación, por cuanto es útil conocer las diferencias y similitudes morfológicas de estas cuatro especies. Examinando 14 medidas morfológicas mediante Análisis Discriminante (LDA) y Análisis de Componentes Principales (PCA), se obtuvo una separación significativa entre las especies, con especial consideración a caracteres asociados con la inserción de las aletas pectorales y pélvicas, y con mediciones respecto a la altura del cuerpo y pedúnculo caudal. También se analizaron 17 hitos anatómicos usando morfometría geométrica mediante PCA y ordenamiento por UPGMA. Los resultados mostraron una separación entre las cuatro especies, encontrando relaciones morfológicas que las distinguen. El ordenamiento por UPGMA coincide parcialmente con los resultados de Campos (1982) y Malabarba (1994), aunque falta incluir a Ch. kiliani, de la cual no existen registros recientes de captura. Los resultados indican que las cuatro especies analizadas muestran claras diferencias morfológicas aplicables a su rápido reconocimiento. Los caracteres analizados dan cuenta de una distinción morfológica que permite su reconocimiento en terreno de forma simple y clara.
\end{abstract}

Palabras clave: Peces, función de placa delgada, análisis discriminante.

\section{SUMMARY}

The freshwater fish of the genus Cheirodon are represented in Chile by five species: Cheirodon pisciculus, Ch. australe, Ch. galusdae, Ch. kiliani and Ch. interruptus, the last introduced accidentally from Argentina. The scarce morphological differentiation as well as the sympatric association of Ch. pisciculus with Ch. interruptus and Ch. galusdae with Ch. australe in part of their distributions makes identification difficult, thus it is useful to know the morphological differences and similarities of these four species. By examining 14 morphological measures using Discriminant Analysis (LDA) and Principal Components (PCA), we found a significant separation between the species, especially in characters associated with the insertion of the pectoral and pelvic fins and measures of body height and caudal peduncle. We also analyzed 17 landmarks with geometric morphometrics using PC and UPGMA grouping. The results showed a separation between the four species, obtaining morphologic relations that distinguish each other. The UPGMA grouping coincided partially with the results of Campos (1982) and Malabarba (1994), although Ch. kiliani should be included, of which there are no recent records of capture. The results show that the four species analyzed have clear morphological differences applicable to their rapid recognition. The characters analyzed produced a morphological distinction that allows their recognition in the field in a clear and simple manner.

KeYwords: Fishes, thin-plate spline, discriminant analysis. 


\section{INTRODUCCIÓN}

Los peces de la familia Characidae comprenden el grupo más abundante y diverso del orden Characiformes, y junto con los peces del orden Siluriformes, dominan la fauna íctica de aguas continentales de Sudamérica (Moyle \& Cech 1995). Esta ictiofauna es altamente endémica y habría evolucionado desde el Terciario, diferenciándose desde las latitudes más tropicales a las más australes (Vila et al. 2006). En Chile, la familia Characidae se encuentra representada por cuatro especies del género Cheirodon, distribuidas de norte a sur: Cheirodon pisciculus Girard, 1854, desde el río Huasco al río Rapel, Ch. galusdae Eigenmann, 1927, desde el río Maule al río Imperial, Ch. australe Eigenmann, 1927, desde el río Valdivia a la Isla de Chiloé, y Ch. kiliani Campos, 1982, especie rara, localizada junto con Ch. australe desde el lago Lanalhue al río Valdivia (Campos 1982, Dyer 2000). Estas especies son típicas de los remansos de los ríos y las zonas costeras bajas de los lagos (Campos 1970), por cuanto se les encuentra preferentemente cerca de la orilla, en la zona superficial de los cursos de agua, sobre sustratos compuestos de fango, arena, grava y/o rocas. Tienden a formar cardúmenes que se establecen en zonas de poca profundidad, en estrecho contacto con la vegetación acuática (Ruiz \& Marchant 2004). Se alimentan principalmente de las comunidades de microalgas epilíticas (auwfuchs) (Vila et al. 1999), aunque estudios de contenido estomacal en especímenes de Ch. galusdae y Ch. pisciculus indican que no se alimentan exclusivamente de perifiton, sino que pueden ser peces típicamente carnívoros, consumiendo diversos taxa de insectos y crustáceos (Fischer 1963, De La Hoz \& Aldunate 1985, Aldunate \& De La Hoz 1993, Ruiz 1993).

Todas las especies de Cheirodon están listadas con problemas de conservación, debido principalmente a la fragmentación del hábitat y modificación de los cursos de agua por efecto antrópico, como son la construcción de represas y canalización, contaminación por residuos industriales y la introducción de especies exóticas (Campos et al. 1998, Habit et al. 2006). De acuerdo al D.S. N $\mathrm{N}^{0} 75 / 2005$, del Ministerio Secretaría General de la Presidencia (Ministerio del Medio Ambiente 2012), el cual oficializa el Reglamento para la Clasificación de Especies Silvestres de acuerdo a las categorías propuestas por la UICN, se determinó por D.S. $\mathrm{N}^{\circ}$ 51/2008 que las especies Ch. australe, Ch. galusdae y Ch. pisciculus se encuentran en categoría "Vulnerable" en todo el país, y Ch. kiliani como "En Peligro y Rara", debido a su distribución restringida y los escasos antecedentes que se disponen (Peredo-Parada et al. 2009).

Además de las cuatro especies nativas antes mencionadas, se lista Ch. interruptus (Jenyns 1842) como el quinto representante del género en Chile. Esta especie fue introducida accidentalmente desde Argentina en la década de los '60 (Arratia 1978, Campos 1982, Malabarba 1994), junto con larvas del pejerrey Odontesthes bonariensis. De hecho, los primeros registros de Ch. interruptus en Chile corresponden al Lago Peñuelas, un embalse artificial donde $O$. bonariensis aparentemente fue cultivado por primera vez (Riegel 1960).

Las cinco especies de Cheirodon forman un grupo monofilético y sus relaciones filogenéticas fueron estudiadas por Malabarba (1994, 1998), cuyos resultados coinciden con el dendrograma de relaciones fenéticas presentado por Campos (1982). No obstante, especies de Cheirodon presentes en la cuenca del río Biobío y entre los ríos Huasco y Aconcagua no han sido satisfactoriamente definidas, pues presentan estados intermedios en los caracteres diagnósticos de Ch. galusdae y Ch australe, y de Ch. pisciculus y Ch. interruptus, respectivamente (Malabarba 1994). Un caso particular es Ch. pisciculus, que se cita con distribución continua desde el río Huasco al río Rapel (Campos 1982, Habit et al. 2006), si bien se sostiene que la introducción de Ch. interruptus y su posterior expansión habría restringido su distribución original (Malabarba 1994). Se requieren nuevos estudios que permitan resolver el rango original de Ch. pisciculus y el actual panorama de la presencia de $C h$. interruptus en los valles de la zona central.

Una de las aproximaciones relevantes para esclarecer la distinción taxonómica de las especies es la morfometría, que estudia la variación de la forma y su covarianza con otras variables (Bookstein 1991). La morfometría clásica o tradicional combina la estadística multivariada y la morfología cuantitativa, analizando la covarianza entre variables como longitudes, anchos y alturas (Adams et al. 2004). La morfometría geométrica, por otro lado, captura información adicional a la morfometría tradicional, pues permite estudiar la geometría de las estructuras de interés (Toro et al. 2010, Zelditch et al 2004). Esta técnica ha incentivado el desarrollo de procedimientos estadísticos que permiten evaluar la cantidad de diferencia entre las conformaciones morfométricas y que facilitan la representación gráfica de los resultados (Bookstein 1991, Rohlf \& Marcus 1993).

El objetivo de este trabajo es encontrar relaciones de forma entre las cuatro especies de Cheirodon mencionadas por Malabarba (1994) que presentan dificultades en su identificación, con una aproximación estadística mediante morfometría tradicional y geométrica. Este estudio tiene el propósito de facilitar la identificación de especímenes en nuevas investigaciones, e.g. aquellas conducentes a esclarecer el patrón actual de distribución e historia biogeográfica del 
género (Dyer 2000), así como otras sobre el género. Además, un correcto reconocimiento en terreno es fundamental si se quiere aprender sobre la ecología del grupo y desarrollar planes de conservación efectivos.

\section{MATERIALES Y MÉTODOS}

\section{MATERIAL BiolóGico}

Se utilizaron 75 individuos de las especies nativas Cheirodon australe, Ch. galusdae y Ch. pisciculus y la especie introducida Ch. interruptus, identificadas según las descripciones de Campos (1982), que comprenden el rango de distribución del género en Chile (Fig. 1, Tabla 1). Se analizaron 28 ejemplares de Ch. australe provenientes de los ríos Petrohué, Cruces y del estero Pichitemuco, 16 individuos de Ch. galusdae de las cuencas de los ríos Mataquito, Biobío, Callecalle y del estero Nonguén, 21 individuos de Ch. pisciculus de los ríos Puchuncaví, Cachapoal y Rapel, y 10 individuos de Ch. interruptus provenientes de los ríos Choapa e Illapel.

El material biológico se obtuvo con pesca eléctrica de bajo impacto durante los años 2008-2009 y fijado con alcohol al $75 \%$.

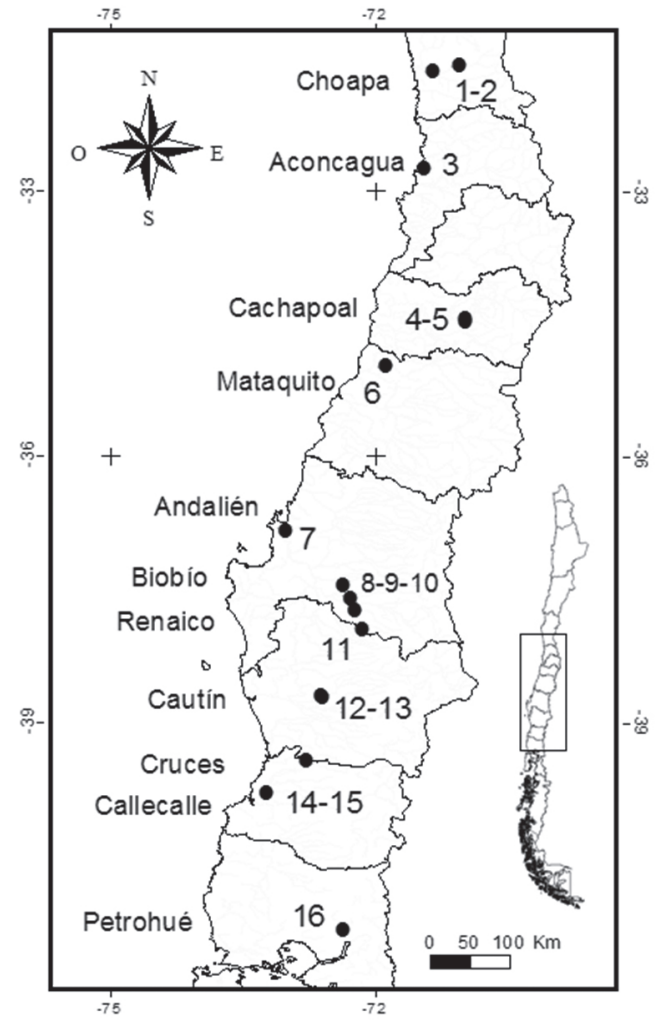

Figura 1. Mapa de Chile con las cuencas y números de los sitios de muestreo; los detalles se muestran en la TABLA 1.

FIGURE 1. Map of Chile with the river basins and the numbers for collection sites; details are shown in Table 1 .
MORFOMETRÍA TRADICIONAL

De cada ejemplar se obtuvieron 14 medidas morfológicas (estandarizadas por la longitud estándar) usando un vernier electrónico de precisión $0,01 \mathrm{~mm}$. Las medidas tomadas son: (1) longitud total (LT), (2) longitud estándar (LS), (3) longitud predorsal (Pre.D), (4) longitud prepectoral (Pre.Pec), (5) longitud prepélvica (Pre.Pel), (6) longitud preanal (Pre.A), (7) altura del cuerpo (Alt.cpo), (8) altura pedúnculo caudal (Alt.PC), (9) longitud pedúnculo caudal (L.PC), (10) longitud preocular (Pre.O), (11) diámetro horizontal del ojo (Diam.O), (12) longitud cabeza (L.cab), (13) altura de la cabeza (Alt.cab) y (14) distancia interorbital (Inter.O) (Fig. 2).

Con la matriz obtenida se realizó un Análisis de Componentes Principales (PCA, en inglés) para establecer los patrones de variación morfológica entre las cuatro especies e identificar aquellas medidas que contribuyen a la distinción en grupos dentro del espacio multivariado. Un valor umbral de $\lambda>$ 1 fue utilizado para determinar los factores relevantes que explican la mayoría de la varianza observada.

Se utilizó un Análisis Discriminante Lineal (LDA, en inglés) para determinar el nivel de discriminación del conjunto de caracteres utilizados y su posibilidad de ser usados en la identificación de ejemplares recolectados desde otras localidades. Tanto el PCA y LDA fueron realizados con el software $\mathrm{R}$ para Windows ( $\mathrm{R}$ Development Core Team 2008).

\section{MORFOMETRÍA GEOMÉTRICA}

Los individuos analizados fueron fotografiados en vista lateral con una cámara de alta resolución. A partir de las imágenes obtenidas se definió la ubicación de 17 hitos anatómicos, atendiendo que la selección describiera aquellas estructuras de interés para identificar externamente los individuos: (1) hocico, (2) proyección anterior de la primera abertura nasal, (3) supraoccipital, (4) inicio y (5) término de la aleta dorsal, (6) inicio de la aleta adiposa, (7) extremo dorsal y (8) ventral del pedúnculo caudal, (10) inicio y (9) término de la aleta anal, (12) inicio y (11) término de la aleta pélvica, (13) sínfisis mentoniana, (14) origen aleta pectoral, (15) división opérculo-subopérculo, (16) origen opérculo y (17) centro interior del ojo (Fig. 3). La digitalización de los hitos y el análisis morfométrico se realizaron con los programas tpsUtil, tpsDig2 y tpsRelw (Rohlf 2012).

El análisis de las configuraciones anatómicas definidas por los hitos se realizó con el software Morpheus (Slice 1998). Se realizó una estandarización de los datos (coordenadas) mediante una superimposición por el método generalizado de mínimos cuadrados (GLS, en inglés), el cual descarta las diferencias de tamaño entre los especímenes corrigiendo los efectos de orientación (rotación, traslación y escala) entre hitos (Rohlf \& Slice 1990). Usando los resultados del GLS 
se realizaron una prueba de ANMUDEVA y una Prueba de $t$ para evaluar si existen diferencias significativas entre las configuraciones consenso de cada especie. Adicionalmente, se analizaron las distorsiones parciales de las variables de forma, ilustrando de manera gráfica el grado de deformación necesaria para que hitos de una especie coincidan con los de otra, utilizando el software Morpheus (mediante la función de placa delgada o thin-plate spline, en inglés; Bookstein 1991).

Los resultados de la superimposición por GLS fueron

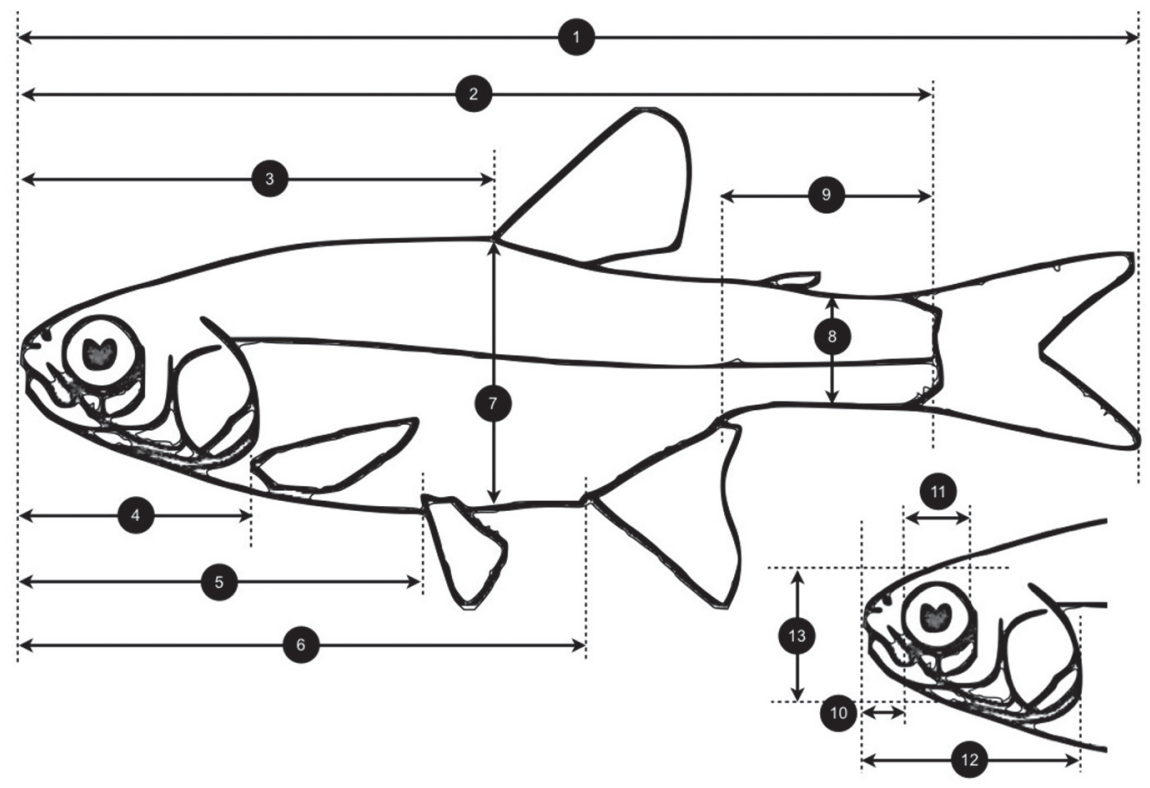

Figura 2. Plano lateral de Cheirodon $s p$. Las medidas morfológicas tomadas se indican en el texto.

Figure 2. Lateral view of Cheirodon sp. The morphological measurements are shown in text.

TABLA 1. Localidades de muestreo. Los códigos corresponden a los números mostrados en la Figura 1. CA, Ch. australe; CG, Ch. galusdae; CI, Ch. interruptus; CP, Ch. pisciculus. La columna localidad indica el curso de agua muestreado y la cuenca correspondiente. Coordenadas UTM Datum WGS84. N, número de individuos examinados.

TABLE 1. Sampling locations. Codes correspond to numbers shown in Figure 1. CA, Ch. australe; CG, Ch. galusdae; CI, Ch. interruptus; $\mathrm{CP}$, Ch. pisciculus. The locality column gives the stream name followed by the river basin. UTM Coordinates Datum WGS84. N, sample sizes examined.

\begin{tabular}{lllllll} 
Código & EsPecie & Localidad & CuENCA & UTM E & UTM N & N \\
\hline 1 & CI & Río Illapel & Choapa & 307215 & 6503816 & 5 \\
2 & CI & Río Choapa & Choapa & 278411 & 6496172 & 5 \\
3 & CP & Estero Puchuncaví & Aconcagua & 272171 & 6373776 & 3 \\
4 & CP & Río Cachapoal & Cachapoal & 319029 & 6185556 & 8 \\
5 & CP & Río Rapel & Cachapoal & 319661 & 6182522 & 10 \\
6 & CG & Río Mataquito & Mataquito & 237794 & 6124121 & 4 \\
7 & CG & Estero Nonguén & Andalién & 677888 & 5921671 & 5 \\
8 & CG & Río Quilque & Biobío & 734963 & 5850360 & 1 \\
9 & CG & Río Biobío & Biobío & 741217 & 5833866 & 3 \\
10 & CG & Río Bureo & Biobío & 745514 & 5820060 & 1 \\
11 & CG & Río Renaico & Renaico & 751145 & 5795507 & 1 \\
12 & CA & Estero Pichitemuco & Cautín & 708184 & 5712705 & 3 \\
13 & CA & Estero Botrolhue & Cautín & 710452 & 5710804 & 1 \\
14 & CA & Río Cruces & Cruces & 691348 & 5631301 & 2 \\
15 & CG & Río Callecalle & Callecalle & 653124 & 5591169 & 1 \\
16 & CA & Río Petrohué & Petrohué & 721045 & 5416986 & 22 \\
\hline
\end{tabular}


utilizados en el Análisis Relative Warp (RWA, en inglés) usando el programa tpsRelw (Rohlf 2012). Con estos resultados se analizaron los patrones de variación entre las especies mediante PCA, y se realizó un agrupamiento por distancias euclidianas utilizando el algoritmo UPGMA (Sneath \& Sokal 1973). Tanto el PCA y el agrupamiento por UPGMA fueron realizados con el software MVSP 3.12c.

\section{RESULTADOS}

MORFOMETRÍA TRADICIONAL

En el análisis de PCA fueron identificados cuatro ejes de variación en total, los cuales explican en conjunto el $82,7 \%$ de la varianza total de los datos. La mayoría de las variables son importantes dentro de los primeros cuatro componentes, destacando la asociación al primer eje (PC1) las variables Pre.Pel, Pre.Pec y L.cab, y al segundo eje (PC2) las variables Diam.O y L.PC. De forma cualitativa, el primer eje posiciona a Cheirodon galusdae en cuadrantes diferentes a Ch. pisciculus (datos no mostrados).

El análisis discriminante mostró que las trazas de los tres discriminantes lineales son 0,75;0,22 y 0,03. Se encontró que Alt.cab, Alt.PC y Alt.cpo son factores importantes dentro del primer discriminante. La Fig. 4 muestra que el Discriminante Lineal 1 (LD1) separa claramente $C h$. interruptus de Ch. pisciculus, y que LD2 muestra diferencias entre Ch. galusdae y Ch. pisciculus.

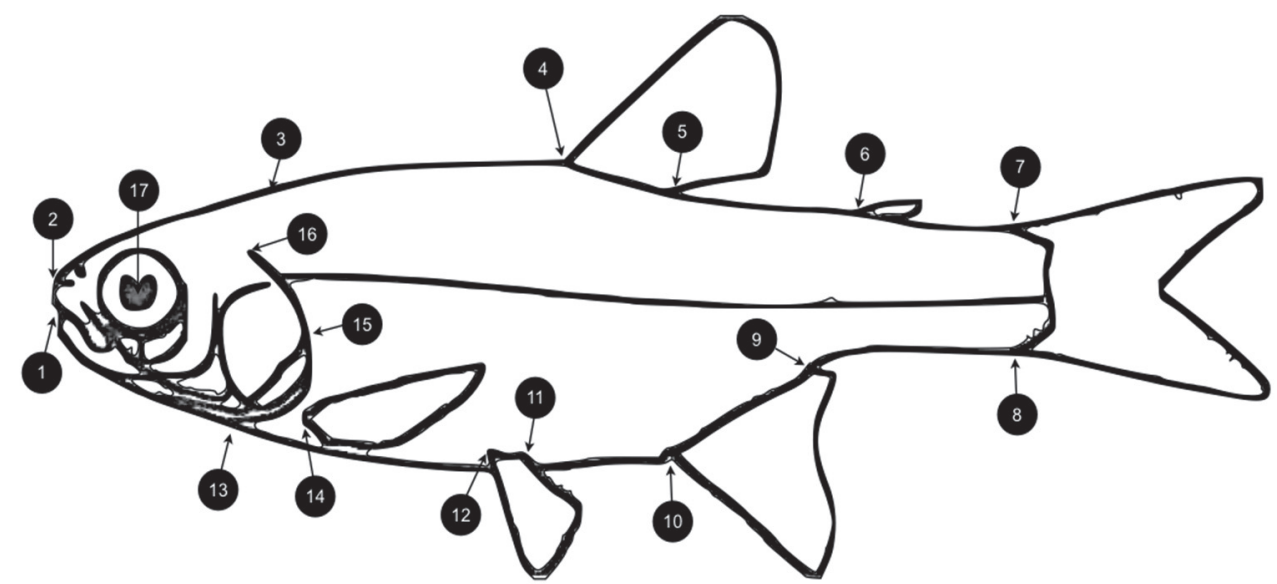

Figura 3. Plano lateral de Cheirodon sp. Los hitos anatómicos definidos se detallan en el texto.

FIgURE 3. Lateral view of Cheirodon sp. The anatomical landmarks are shown in text.

TABLA 2. Caracteres morfológicos que diferencian las especies de Cheirodon estudiadas utilizando relaciones de extensión de las aletas respecto al origen. Se indica el éxito en la matriz de clasificación a partir del LDA.

TABLE 2 . Morphological features that differentiate the species of Cheirodon using the extension of fins from the origin. It also shows the success of classification matrix from LDA.

\begin{tabular}{lcccc} 
CARACTERística & Ch. australe & Ch. galusdae & Ch. pisciculus & Ch. interruptus \\
\hline $\begin{array}{l}\text { El rayo más largo extendido } \\
\text { horizontalmente de la aleta pectoral } \\
\text { alcanza el origen de la aleta pélvica }\end{array}$ & Sí & Sí & No & Sí \\
$\begin{array}{l}\text { La aleta pélvica extendida } \\
\text { horizontalmente alcanza el origen de } \\
\text { la aleta anal }\end{array}$ & No & Sí & No & No \\
$\begin{array}{l}\text { Perfil de la aleta anal al comparar } \\
\text { el rayo más largo extendido con el } \\
\text { último }\end{array}$ & transversal & vertical & vertical & vertical \\
\hline $\begin{array}{l}\text { Porcentaje de éxito de la matriz de } \\
\text { clasificación a partir del LDA }(\%)\end{array}$ & 79 & 81 & 95 & 80
\end{tabular}



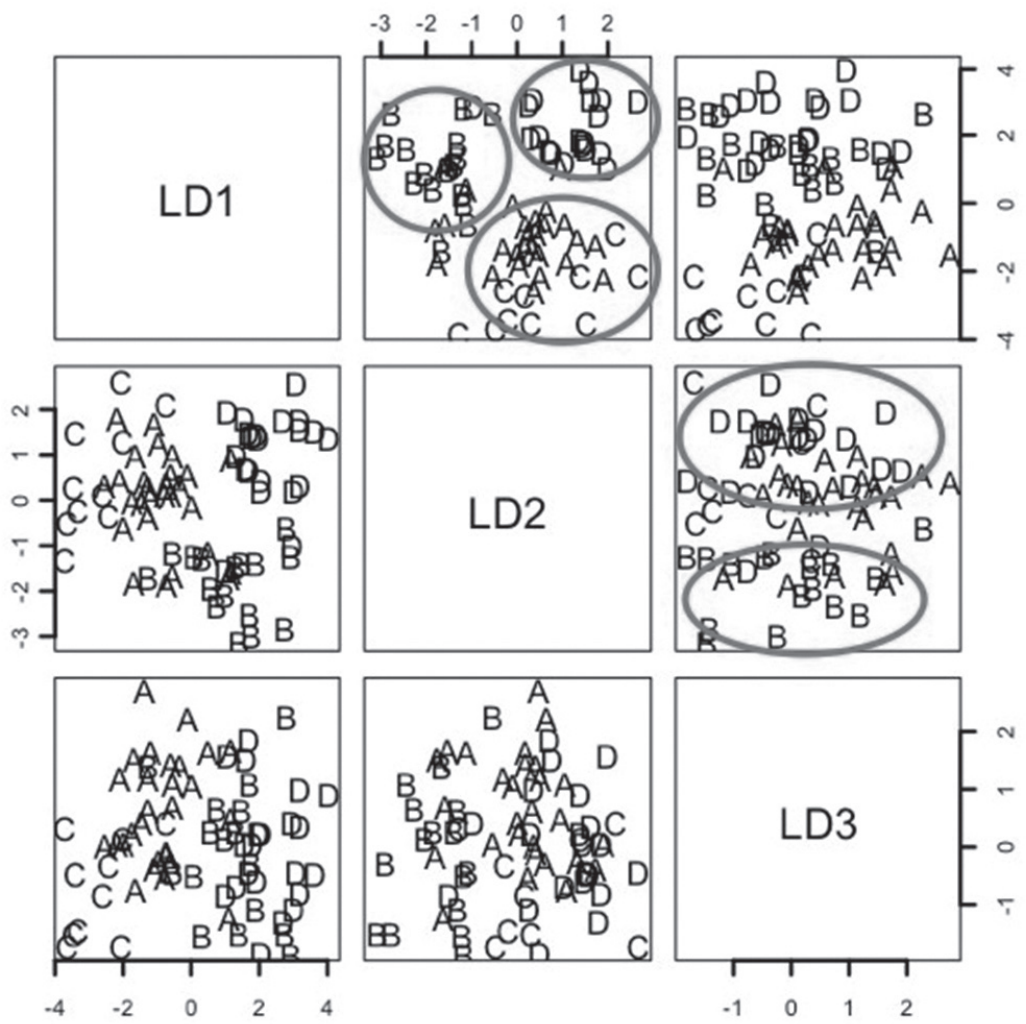

Figura 4. Análisis discriminante lineal para las especies de Cheirodon. A, Ch. australe; B, Ch. galusdae; C, Ch. interruptus; D, Ch. pisciculus. Los círculos indican las agrupaciones entre especies.

Figure 4. Linear discriminant analysis for the species of Cheirodon. A, Ch. australe; B, Ch. galusdae; C, Ch. interruptus; D, Ch. pisciculus. Groups between species are shown in circles.

Estos resultados indican que los caracteres asociados a la disposición de las aletas y con proporciones relacionadas con medidas de la cabeza y el pedúnculo caudal son relevantes para la diferenciación.

Ninguno de los dos análisis estadísticos permitió diferenciar los individuos de Ch. australe del resto de especies, obteniendo además el menor porcentaje de éxito en la clasificación de los individuos por especie (Tabla 2).

\section{MORFOMETRÍA GEOMÉTRICA}

Los resultados del ANMUDEVA sobre los datos agrupados por especie indicaron que existen diferencias significativas entre los grupos $(\mathrm{p}<0,01)$. La prueba de $\mathrm{t}$ también mostró diferencias altamente significativas entre las especies $(p<$ 0,001 para todos los casos).

El análisis de las diferencias morfométricas también puede ser abordado de manera gráfica mediante la función de placa delgada (TPS, en inglés), la cual permite observar el grado de deformación de la conformación morfométrica de una especie respecto a otra (Bookstein 1991, Toro et al. 2010). Para todas las especies se obtuvo que los caracteres relevantes para su caracterización son los mismos sugeridos por la morfometría tradicional: la disposición de las aletas y relaciones generales de la cabeza, altura del cuerpo y del pedúnculo caudal. En la Fig. 5 se muestran las grillas de transformación entre las especies.

Comparativamente, se observa que Cheirodon australe presenta una deformación de la cabeza y altura del cuerpo mayores que Ch. galusdae, por cuanto esta última suele presentarse como un individuo más alargado. No presentan diferencias respecto a la extensión y altura del pedúnculo caudal, aunque en Ch. galusdae el pedúnculo puede presentarse más deformado en la porción ventral. No obstante, este carácter no fue observado en todos los especímenes. Una diferencia leve es la posición del origen de la aleta adiposa, la cual es opuesta al origen del último radio de la aleta anal en Ch. australe, y en Ch. galusdae se presenta contraria al pedúnculo caudal, levemente alejada del término de la aleta anal. En Ch. galusdae se observa 
que la distancia entre la inserción de la aleta pectoral y la pélvica es mayor que en Ch. australe, por cuanto al extender horizontalmente la aleta pectoral esta no alcanza el origen de la pélvica. En Ch. australe este espacio es menor o bien puede no presentarse, coincidiendo el rayo más largo de la pectoral con el origen de la aleta pélvica.

Entre Ch. interruptus y Ch. pisciculus se observa que la primerapresentaunadeformacióncorporal significativamente mayor que Ch. pisciculus. Esto produce que los ejemplares de Ch. interruptus sean descritos como peces más altos y menos alargados que las especies de Cheirodon chilenas. Además, el pedúnculo caudal se inicia más adelante que en Ch. pisciculus, lo que contribuye a que se observe como una especie con una menor deformación relativa a los caracteres corporales generales. Entre Ch. interruptus y Ch. pisciculus casi no hay diferencias entre la inserción de la aleta pectoral, pero sí difieren significativamente en el origen de la aleta pélvica, en que $C h$. pisciculus presenta una inserción de la pélvica cercana a la porción central del cuerpo, opuesta a la aleta dorsal, mientras que en Ch. interruptus se presenta más cercana a la aleta pectoral. Esto produce que se observe un espacio entre el rayo más largo extendido de la aleta pectoral y el origen de la pélvica en Ch. pisciculus, mientras que en Ch. interruptus la aleta pectoral usualmente sobrepasa

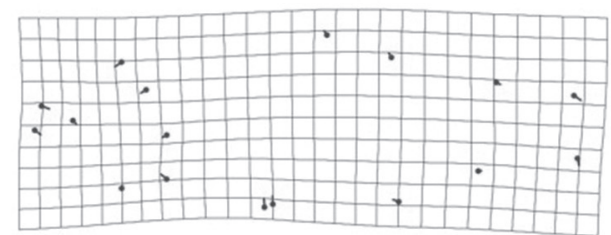

Ch. australe - Ch. galusdae

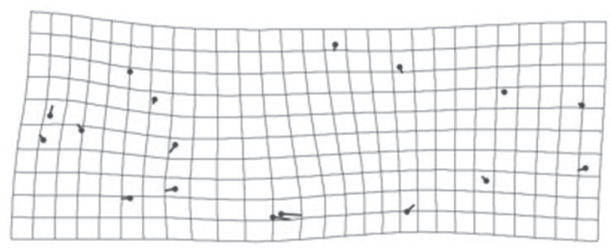

Ch. australe - Ch. pisciculus

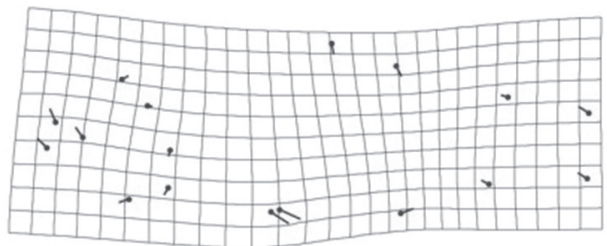

Ch. galusdae - Ch. pisciculus el origen de la pélvica. Así también, este mismo espacio puede observarse en Ch. pisciculus entre la aleta pélvica y el origen de la aleta anal, mientras que en Ch. interruptus este intervalo es menor o puede no existir, coincidiendo el rayo más largo de la pélvica extendido con la inserción de la aleta anal.

Las comparaciones entre aquellas especies con distribución alopátrica (e.g. Ch. australe y Ch. pisciculus) responden a un reconocimiento basado en las proporciones asociadas al origen de las aletas pélvica y anal respecto de la extensión horizontal de las aletas pectoral y pélvica, respectivamente (Fig. 5, Tabla 2).

Las coordenadas generadas luego de la superimposición por GLS fueron analizadas mediante un PCA (RWA), cuyos resultados pueden ser presentados en función de los componentes principales para agrupar los individuos por grupo morfológico. Los primeros 10 componentes explicaron el 90,05\% de la varianza observada. No obstante, no se observaron asociaciones entre los primeros dos componentes, encontrando una escasa o bien nula distinción de especies, por cuanto si bien se observaron diferencias significativas en los análisis de ANMUDEVA y Prueba de t, estas no se reflejan en el análisis gráfico del RWA.

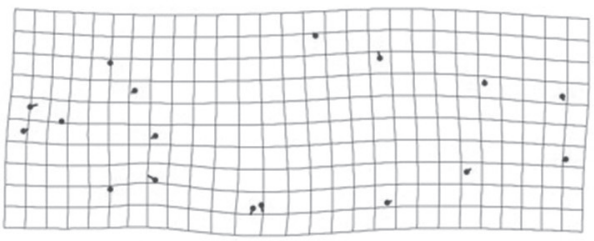

Ch. australe - Ch. interruptus

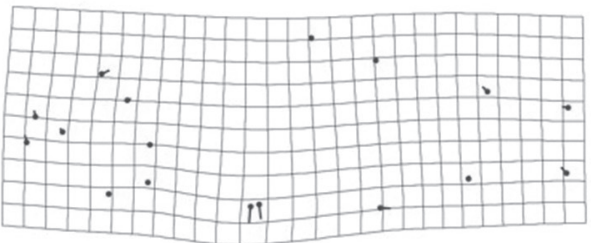

Ch. galusdae - Ch. interruptus

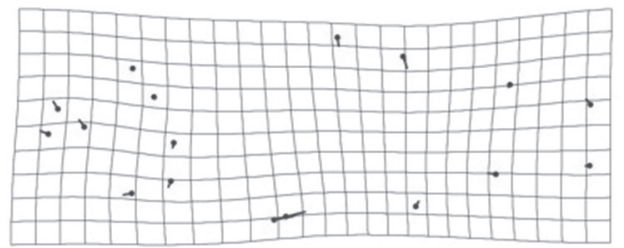

Ch. interruptus - Ch. pisciculus

FIgURA 5. Grillas de transformación comparativas entre las especies estudiadas.

FIGURE 5. Comparative transformation grids between studied species. 
Con los primeros 10 componentes principales del RWA se realizó un agrupamiento por distancias euclidianas utilizando el algoritmo UPGMA. Este agrupamiento muestra resultados parcialmente similares a los encontrados por Campos (1982), excluyendo del análisis a Ch. kiliani (Fig. 6). Se observa un primer grupo constituido por Ch. australe y Ch. interruptus, los cuales comparten la mayoría de los caracteres analizados en este trabajo. La siguiente especie asociada a este grupo es Ch. galusdae, y más distante se encuentra $C h$. pisciculus. Aun cuando las agrupaciones y distancias no son significativas, destaca la separación entre las especies analizadas en este estudio que tienen dificultades en su identificación: Ch. pisciculus y Ch. interruptus, y Ch. australe y Ch. galusdae (Dyer 2000, Habit et al. 2006).
En la Tabla 2 se resumen las características importantes desprendidas de la morfometría tradicional y geométrica para un fácil reconocimiento de las especies del género Cheirodon de Chile. Todas las especies pueden ser eventualmente discriminadas usando estas características, con excepción de Ch. australe y Ch. interruptus que comparten los caracteres. Para estas dos especies los análisis estadísticos no son concluyentes, y por el momento sólo se sugiere la comparación del perfil de la aleta anal, en que en Ch. interruptus el rayo más largo alcanza al último rayo de la anal, formando un perfil vertical, mientras que en $C h$. australe el rayo más largo no alcanza al último y se observa un perfil diagonal (Campos 1982).
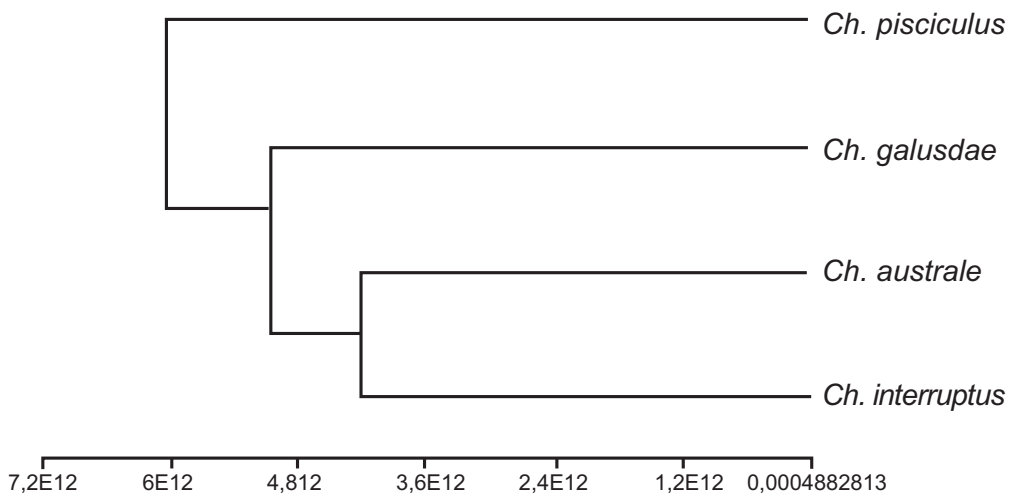

Distancia Euclidiana

Figura 6. Agrupamiento por UPGMA utilizando el individuo consenso de la morfometría geométrica de las especies analizadas en este estudio.

FIGURE 6. UPGMA clustering using individual consensus from geometric morphometric analysis.

\section{DISCUSIÓN}

Si bien las especies de Cheirodon en Chile tienen distribuciones geográficas continuas y tendencia a la alopatría, presentan una similitud interespecífica que impide la presencia de caracteres fenotípicos de diagnóstico que permitan clasificar las poblaciones con claridad. Como ya lo manifestara Campos (1982), los caracteres de diagnóstico son una combinación de características que se asocian en cada especie con la presencia o ausencia de una de ellas. Tanto para Campos (1982) y Malabarba (1994) la combinación de caracteres se hace difusa en algunas poblaciones.

Los estudios referidos a la discriminación de especies del género Cheirodon asocian el número de cúspides de los dientes como un carácter significativo. No obstante, las cúspides en los dientes varían tanto inter- como intraespecíficamente, por cuanto suele llevar a error una identificación basada en esta característica (Campos 1982). Un caso similar ocurre con los rayos prorrecurrentes de la porción dorsal y ventral del pedúnculo caudal, los cuales presentan un escaso desarrollo y su número es semejante en todas las especies, correspondiendo además a un carácter de dimorfismo sexual (Arratia 1987). No obstante, en especímenes en buen estado suele ser un carácter de diagnóstico la longitud del pedúnculo caudal que es ocupada por los rayos prorrecurrentes. Estos antecedentes indican que la discriminación de especies se fundamenta más en caracteres merísticos que morfológicos, cuya aplicación requiere conocimiento y experiencia en el tratamiento de los especímenes, dificultando la identificación a personas ajenas a la ictiología. En efecto, Campos (1982) insiste en que la diferenciación depende en primer lugar de caracteres merísticos y posteriormente en modificaciones corporales que parecen más estables. 
El presente estudio pretende reanalizar varias de las características examinadas por Campos (1982) y Malabarba (1994) utilizando morfometría tradicional, y encontrar relaciones de forma utilizando morfometría geométrica, de reciente aplicación en Chile para la fauna íctica (e.g. Pardo 2002, Pardo et al. 2005, Gacitúa et al. 2008), con el objetivo de facilitar el reconocimiento de las especies de Cheirodon en trabajos de campo.

En los análisis realizados se encontró que las características asociadas a la posición de las aletas, sus relaciones respecto a las inserciones, y caracteres relacionados a la forma del cuerpo son útiles para la identificación de las especies de Cheirodon. Tanto la longitud prepélvica, altura del cuerpo, longitud del pedúnculo caudal, longitud de la cabeza, son caracteres relevantes para la descripción de los especímenes, aun cuando la descripción puede reducirse a una serie de características de fácil observación (Fig. 7). Estas regiones corporales fueron identificadas tanto con la morfometría tradicional y geométrica.

Las relaciones entre las especies respecto a los caracteres analizados son relevantes en tanto coinciden parcialmente con las relaciones presentadas por Campos (1982) y Malabarba (1994). Se encontró un primer grupo conformado por Ch. australe y Ch. interruptus, los cuales comparten una serie de características, pero que pueden ser fácilmente distinguidos por la altura corporal y el perfil de la aleta anal respecto al rayo más largo (Campos 1982). A continuación se ubica $C h$. galusdae como la especie más cercana a este grupo, cuyas características principales son la ausencia del espacio entre el rayo más largo de la aleta pectoral y el origen de la aleta pélvica, y la ausencia del espacio entre el rayo más largo de la pélvica y el origen de la aleta anal. Más distante se encuentra Ch. pisciculus, que presenta ambos espacios entre los rayos extendidos de la pectoral (y pélvica) respecto al origen de la pélvica (y anal).

Los análisis estadísticos separaron claramente las poblaciones de Ch. galusdae y Ch. pisciculus. Si bien estas especies se distribuyen de forma continua en sentido latitudinal, no presentan dificultad en su identificación en razón de sus aparentemente diferentes localidades tipo (Vila et al. 2006). No obstante, los resultados morfométricos permiten establecer distinciones asociadas a la posición en la inserción de las aletas y relaciones entre la cabeza y el pedúnculo caudal respecto a la longitud estándar, cuyas comparaciones pueden realizarse indistintamente en cada individuo y no requieren ser presentadas mediante la consideración de los holotipos del resto de especies.

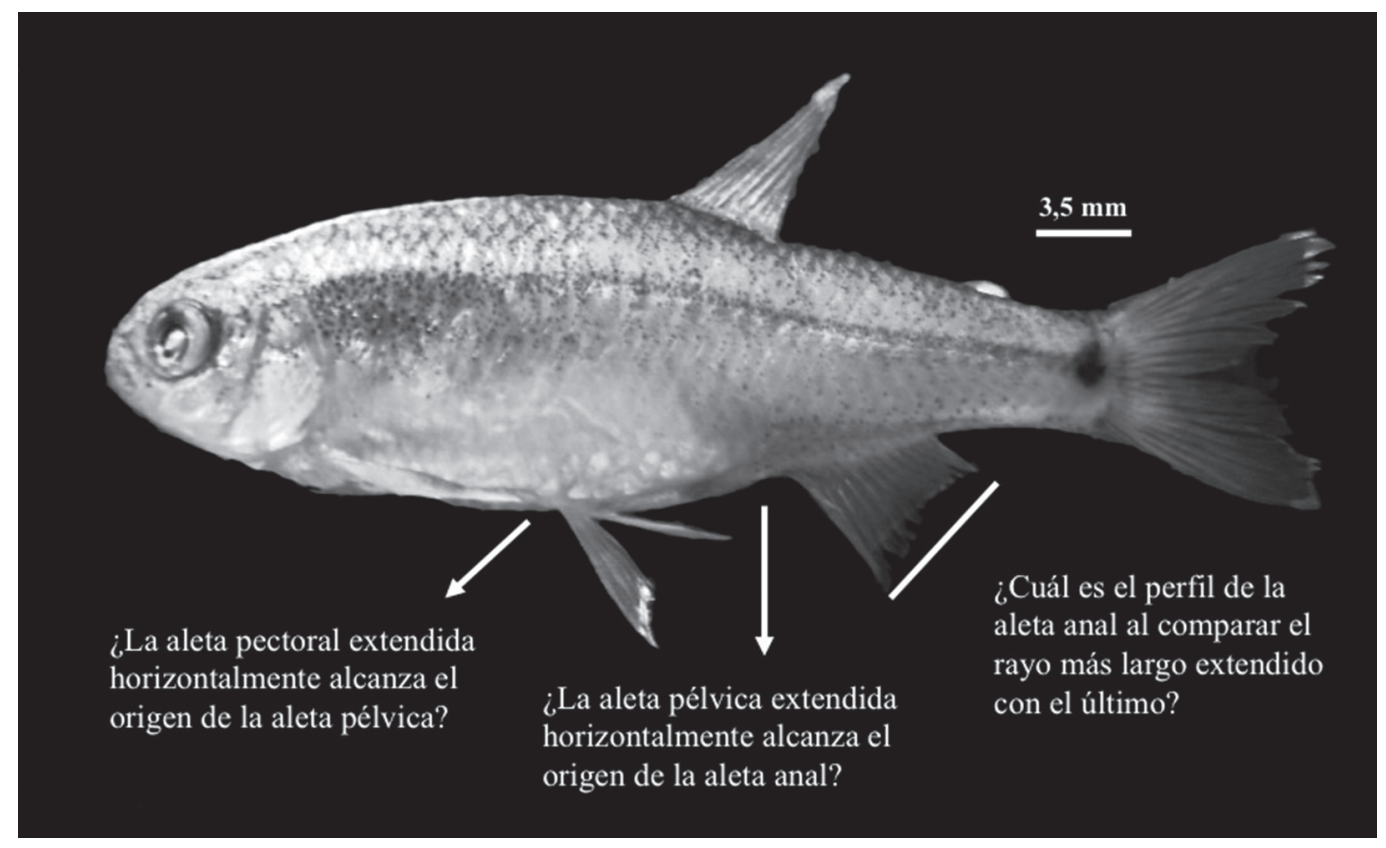

FIGURA 7. Representación esquemática de los caracteres relevantes para un fácil reconocimiento de las especies de Cheirodon; las respuestas para cada especie se muestran en la TABLA 2.

FIGURE 7. Schematic representation of the relevant characters for easy recognition of species of Cheirodon; answers for each species are shown in Table 2. 
El ordenamiento de las especies a partir de los datos de morfometría geométrica no mostraron la misma organización presentada por Campos (1982), en que se observa un grupo distinto conformado por Ch. galusdae y Ch. pisciculus, probablemente debido a la ausencia de ejemplares de Ch. kiliani. Individuos de esta última especie no fueron analizados por los escasos antecedentes que se disponen, considerando la ausencia de registros de captura desde su descripción (Möller 2008, Peredo-Parada et al. 2009). Por tanto, se sostiene que los caracteres de diagnóstico de $C h$. kiliani no han sido correctamente evaluados y probablemente presenten estados difusos con Ch. australe. Sin embargo, el ordenamiento presentado en este estudio es informativo desde la perspectiva biogeográfica, en tanto se observa una clasificación inclusiva partiendo por Ch. pisciculus hasta Ch. australe en relación a sus rangos de distribución, excluyendo a Ch. interruptus que comparte su distribución con Ch. pisciculus y además no es nativa.

Las relaciones presentadas en este trabajo responden a una recomendación para facilitar la identificación de estas especies, en especial para su uso en campo, debido a que todos los caracteres que sirven para su diferenciación corresponden a caracteres externos (Fig. 7).

Si bien las diferencias encontradas no responden a un patrón asociado a la descripción de las cuencas hidrográficas donde habitan las especies (Vila et al. 1999), por cuanto las relaciones presentadas no forman parte de una descripción de diferenciación histórica, estas son útiles para su reconocimiento tanto en trabajos científicos como en líneas bases o estudios de impacto ambiental. Se sugiere, como continuación de este estudio, ampliar la caracterización y revalidación de las especies de Cheirodon desde una aproximación histórica y espacial.

\section{AGRADECIMIENTOS}

Los autores agradecen a GESAM Consultores Ltda. por facilitar parte de las muestras analizadas en este artículo. DV agradece financiamiento de Fondecyt 1100341, Instituto de Ecología y Biodiversidad, ICM P05-002 y Fondo Basal PFB-23 (Conicyt, Chile).

\section{REFERENCIAS}

Adams, D.C., Rohlf, F.J. \& Slice, D.E. 2004. Geometric morphometrics: ten years of progress following the 'revolution'. Italian Journal of Zoology 71: 5-16.

Aldunate, R. \& De La Hoz, E. 1993. Diversidad trófica de Cheirodon pisciculus G. (Ostariophysi: Characidae): ¿consecuencia de una versatilidad del mecanismo alimentario? Revista Chilena de Historia Natural 66: 177-184.
Arratia, G. 1978. Comentario sobre la introducción de peces exóticos en aguas continentales de Chile. Ciencias Forestales 1: 21-30.

Arratia, G. 1987. Sexual dimorphism in the caudal skeleton of Cheirodon (Characidae, Teleostei). Cybium 11: 375-387.

Bookstein, F.L. 1991. Morphometric tools for landmark data: geometry and biology. Cambridge University Press, Cambridge.

CAmpos, H. 1970. Introducción de especies exóticas y su relación con los peces de agua dulce de Chile. Noticiario Mensual Museo Nacional de Historia Natural, Chile 14: 3-9.

Campos, H. 1982. Sistemática del género Cheirodon (Pisces: Characidae) en Chile con descripción de una nueva especie. Análisis de multivarianza. Studies on Neotropical Fauna and Environment 17: 129-162.

Campos, H., Dazarola, G., Dyer, B., Fuentes, L., Gavilán, J.F., Huaquín, L., Martínez, G., Meléndez, R., Pequeño, G., Ponce, F., Ruiz, V.H., Siefeld, W., Soto, D., Vega, R. \& VILA, I. 1998. Categorías de conservación de peces nativos de aguas continentales de Chile. Boletín del Museo Nacional de Historia Natural 47: 101-122.

De La Hoz, E. \& Aldunate, R. 1985. Mecanismo mandibular en Cheirodon pisciculus Girard, 1854 (Ostariophysi: Characidae). Revista Chilena de Historia Natural 58: 139155.

Dyer, B. 2000. Systematic review and biogeography of the freshwater fishes of Chile. Estudios Oceanológicos (Chile) 19: 77-98.

Fischer, W. 1963. Die fisches des Brackwassergebietes Lenga bei Concepción (Chile). Internationale Revue der gesamten Hydrobiologie und Hydrographie 48: 419-511.

Gacitúa, S., Oyarzún, C. \& Veas, R. 2008. Análisis multivariado de la morfometría y merística del robalo Eleginops maclovinus (Cuvier, 1830). Revista de biología marina y oceanografía 43: 491-500.

Habit, E., Dyer, B. \& Vila, I. 2006. Estado de conocimiento de los peces dulceacuícolas de Chile. Gayana (Concepción) 70: 100-113.

Malabarba, L.R. 1994. Sistemática e filogenia de Cheirodontinae (Ostariophysi: Characiformes: Characidae). Tesis Doutorado, Universidade de Sao Paulo, Brasil, 287 pp.

Malabarba, L.R. 1998. Monophyly of the Cheirodontinae, characters and major clades (Ostariophysi: Characidae). En: Phylogeny and classification of Neotropical fishes. Malabarba L.R., R.E. Reis, R.P. Vari, Z.M. Lucena, C.A.S. Lucena (Eds). Porto Alegre, Edipucrs, pp. 193-233.

Ministerio Del Medio Ambiente. 2012. Clasificación de las especies según su estado de conservación. URL: http:// www.mma.gob.cl/clasificacionespecies/ Accesado: Junio 4, 2012.

Möller, P. 2008. Bibliografía sobre peces de aguas continentales de Chile. Gestión Ambiental (Valdivia) 16: 67-92.

Moyle, P.B. \& CeCH JR, J.J. 1995. Fishes. An Introduction to Ichthyology. Third Edition. Prentice Hall, Inc. USA. 612 pp.

Pardo, R. 2002. Diferenciación morfológica de poblaciones de Trichomycterus areolatus Valenciennes 1846 (Pisces: Siluriformes: Trichomycteridae) de Chile. Gayana 66: 203-205.

Pardo, R., Scott, S. \& Vila, I. 2005. Análisis de formas en especies 
chilenas del género Trichomycterus (Osteichthyes: Siluriformes) utilizando morfometría geométrica. Gayana 69: 180-183.

Peredo-Parada, M., Martínez-Capel, F., Garófano-Gómez, V., Atenas, M. \& Riestra, F. 2009. Base de datos ecohidrológica de los ríos de Chile: una herramienta de gestión para los ecosistemas acuáticos. Gayana 73: 119-129.

R Development Core Team. 2008. R: A language and environment for statistical computing. R Foundation for Statistical Computing, Vienna, Austria. URL: http://www.R-project. org/

RiEgel, H. 1960. Observaciones sobre la fauna ictiológica de las aguas dulces chilenas. Actas y Trabajos del Primer Congreso Sudamericano de Zoología, La Plata, Tomo I: 141-144.

RoHLF, F.J. 2012. SB Morphometrics. Department of Ecology and Evolution, State University of New York at Stony Brook. URL: http://life.bio.sunysb.edu/morph/ Accesado: Junio 4, 2012.

Rohlf, F.J. \& Slice, D.E. 1990. Extensions of the Procrustes method for the optimal superimposition of landmarks. Systematic Biology 39: 40-59.

RohlF, F.J. \& MARCus, L.F. 1993. A revolution in morphometrics. Trends in Ecology and Evolution 8: 129-132.
RuIZ, V.H. \& MARCHANT, M. 2004. Ictiofauna de aguas continentales chilenas. Primera edición. Universidad de Concepción, Concepción. 356 pp.

RuIz, V.H. 1993. Ictiofauna del Río Andalién. Gayana 57: 109278.

SLICE, D.E. 1998. Morpheus et al.: software for morphometric research. Recent beta revision. Stony Brook, New York. Department of Ecology and Evolution, State University of New York.

Toro, I.M., Manriquez, S.G. \& Suazo, G.I. 2010. Morfometría geométrica y el estudio de las formas biológicas: de la morfología descriptiva a la morfología cuantitativa. International Journal of Morphology 28: 977-990.

Sneath, P.H. \& Sokal, R.R. 1973. Numerical Taxonomy San Francisco: Freeman.

Vila, I., Fuentes, L. \& Contreras, M. 1999. Peces límnicos de Chile. Boletín del Museo Nacional de Historia Natural 48: 61-75.

Vila, I., Veloso, A., Schlatter, R. \& Ramírez, C. 2006. Macrófitas y vertebrados de los sistemas límnicos de Chile, Primera Edición. Editorial Universitaria, Santiago. 187 pp.

Zelditch, M.L., Swiderski, D.L., Sheets, H.D. \& FinK, W.L. 2004. Geometric Morphometrics for Biologists: A Primer. Elsevier Academic Press. 443 pp.

Recibido: 23.03.12

Aceptado: 27.06 .12 